Materials and technological processes for High-Gradient accelerating structures: new results from mechanical tests of an innovative braze-free cavity.

To cite this article: V.A. Dolgashev et al 2020 JINST 15 P01029

View the article online for updates and enhancements.

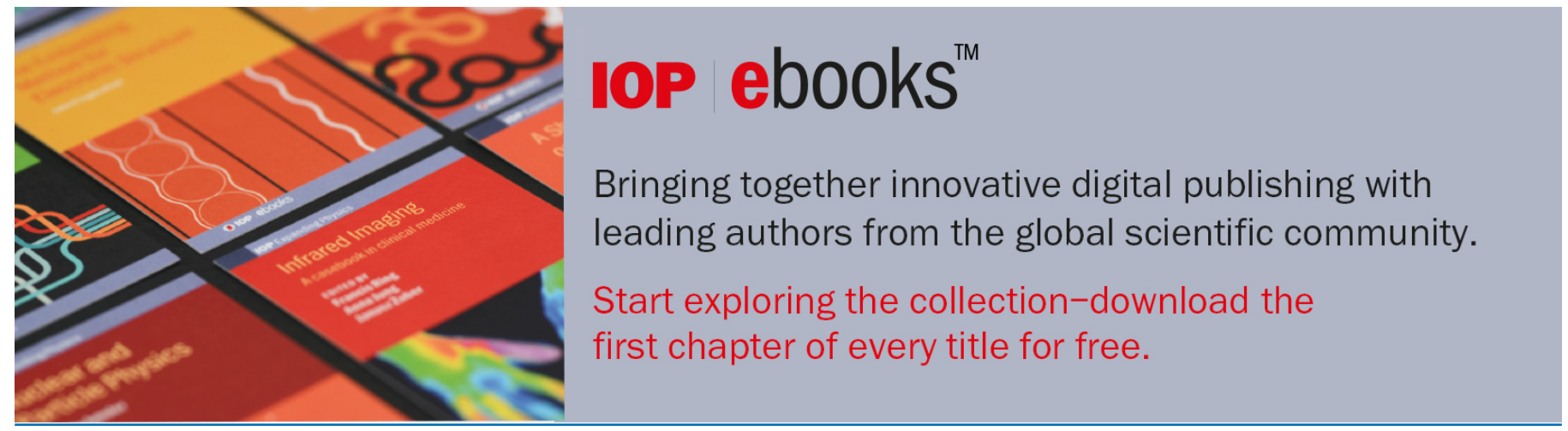




\section{Materials and technological processes for High-Gradient accelerating structures: new results from mechanical tests of an innovative braze-free cavity.}

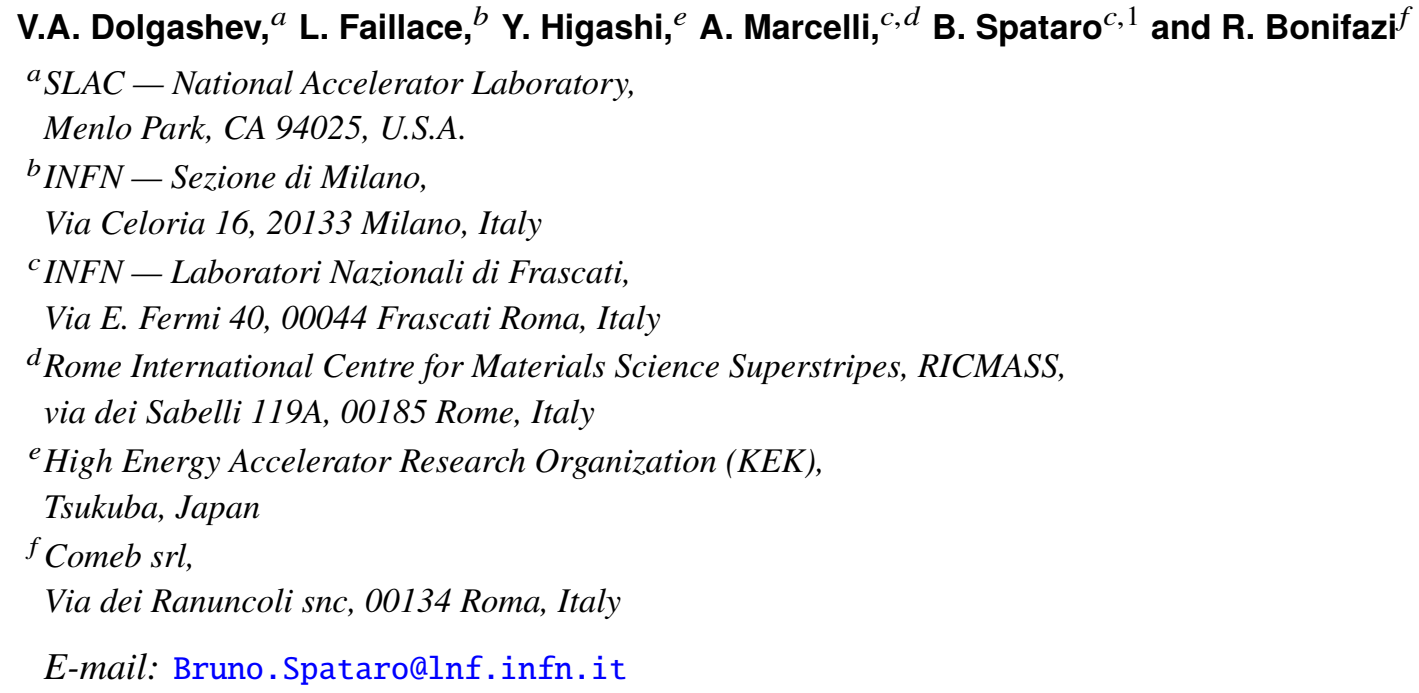

Abstract: Pure oxygen-free high-conductivity copper is a widely used material for manufacturing accelerating cavities working at room temperature. Several studies attempted to explain limitations associated with the maximum allowed field gradients and the behaviour of vacuum RF breakdown in copper accelerating structures through generation and movement of dislocations under stresses associated with RF electric and magnetic fields. Pure copper and also copper alloys undergo mechanical and thermal treatments to be hardened and strengthened during manufacturing, although their mechanical properties significantly change after heating above $590^{\circ} \mathrm{C}$. High temperature brazing and diffusion bonding are assembly methods widely used to manufacture ultra-high vacuum accelerating devices. However, these processes, occurring at about $800-1000^{\circ} \mathrm{C}$, significantly affect the mechanical properties of copper and copper alloys. We present here a novel Tungsten Inert Gas welding procedure, which is fast and keeps the high-gradient surfaces of the cavity and other components well below the copper annealing temperature. This process may be successfully used to manufacture copper-based accelerating components. This technology preserves the hardness and cleanliness of copper in order to achieve the maximum accelerating gradient.

KeYwords: Accelerator Applications; Accelerator Subsystems and Technologies

\footnotetext{
${ }^{1}$ Corresponding author.
} 


\section{Contents}

1 Introduction 1

2 Cavity construction 2

3 TIG welding process 3

4 Optimization of the welding tooth 3

5 The welding set-up 5

6 Temperature reading 5

7 Welding of the final prototype $\quad 8$

8 Conclusions $\quad 8$

\section{Introduction}

There is a strong demand for accelerating structures able to achieve higher gradients and more compact footprints for the next generation of linear accelerators [1], for research, industrial and medical applications $[2,3]$. The project presented herein is the result of a continuous, decade-long collaboration involving the laboratories of KEK in Japan, SLAC in U.S.A. and INFN in Italy.

Recent experiments [4-6] have demonstrated that structures made out of hard copper alloys can achieve reliable accelerating gradients higher than $150 \mathrm{MV} / \mathrm{m}$ in X-Band with lower RF breakdown rates (BDR) below $10^{-2} / \mathrm{pulse} / \mathrm{m}$ breakdown probability, with respect to annealed ones. Commonly used methods for joining parts of accelerating structures are high temperature brazing and diffusion bonding, which are performed at temperatures of $800-1000^{\circ} \mathrm{C}$. This high temperature anneals the metal and makes it soft. Although brazed bonds have good electrical, thermal and vacuum properties, experimental results with hard copper cavities showed that hard materials sustain higher accelerating gradients for the same breakdown rate, with copper-silver having the best performance. Complementary to RF breakdown tests, studies of RF induced pulse-surface heating have shown that hard, non-annealed material alloys such as $\mathrm{CuAg}, \mathrm{CuCr}$ and/or $\mathrm{CuZr}$ exhibited significantly less damage than annealed copper samples [9]. High power tests of hard $\mathrm{CuAg} \mathrm{X-Band} \mathrm{structures}$ allowed to achieve gradients up to $200 \mathrm{MV} / \mathrm{m}$ at $10^{-3} / \mathrm{pulse} / \mathrm{m}$ breakdown probability.

We have conducted a systematic study of metal damage due to RF induced pulse surface heating [9]. In this work we have measured hardness of different samples from 10 to 85 HR15T (see figure 54 of the [9]). The results of this study were used as a guidance for our high gradient tests. For copper and copper silver we have found that cavities made of harder metals have lower breakdown rate then ones made of the softer metals. Other metals did not have superior performance to 
copper independent on their high values of initial hardness, which is not surprising given complexity of the physics of vacuum RF breakdown. There is another difficulty of correlating initial hardness of the metals and their breakdown performance. During the measurements of the breakdown rates the cavities are "conditioned" with millions of RF pulses and thousands of breakdowns. This conditioning changes ultimate hardness of the metal and distribution of the hardness over the cavity surface. We note that the study of the effect of metal hardness on breakdown performance is continuing both with RF and DC experiments [10,11].

Positive effects of hardness on RF BDR are also supported by recent cryogenics tests performed at SLAC [12]. Indeed, by decreasing crystal mobility and increasing yield stress, the breakdown rate is reduced for the same gradient. We want to achieve this goal by cooling a cavity below $45 \mathrm{~K}$. At this temperature, the copper HB index (around 55/60 at room temperature), increases by about 25\%, which corresponds to a yield strength raise of nearly $80 \mathrm{MPa}$ [13]. These cryo normal-conducting accelerating cavities hold the absolute record for X-band accelerating structures of $\sim 250 \mathrm{MV} / \mathrm{m}$ $\left(0.5 \mathrm{GV} / \mathrm{m}\right.$ peak surface) at $45 \mathrm{~K}$ with $10^{-3} /$ pulse/m breakdown probability.

All these beneficial properties of hard copper-based structures need to be preserved during the manufacturing process. We have chosen to perform the Tungsten Inert Gas (TIG) welding of the outer surfaces of novel type of accelerating structures, which allows to build practical multi-cell cavities up to the order of a meter. In figure 1, it is shown the first 3-cell prototype that was fabricated at INFN [14] and shipped to the SLAC national accelerator laboratory in the U.S.A. for high RF power tests. The TIG process assures a steady vacuum envelope and robust mechanical assembly. Nevertheless, it can modify the structure geometry and the products of the weld will contaminate and destroy the performance. Since in our case, the TIG welding is the final step of the machining process, we have developed a technology process in order to control the cavity geometrical dimensions as well the cleanliness preservation.

Here, we present this technology together with the mechanical tests performed on cavity samples, made of two or three cells (based on the first prototype geometry), for the temperature and stress-related characterization and corresponding cleanliness of the structure.

\section{Cavity construction}

In figure 1 it is shown the solid model of the first prototype of the 3-cell accelerating X-band structure, that we termed the "Squeeze cavity", which shows the proposed clamping system, which is achieved by means of special and proprietary screws ( 2 in the figure). Thanks to this innovative approach, it is possible to obtain perfect RF contacts between the cells and the desired quality factor without using any high-temperature bonding process. Two vacuum regions or chambers are present in the structure. The primary RF vacuum chamber (1) is the volume inside the highgradient accelerating cells, where the RF power builds up the electromagnetic field. This chamber is connected to a vacuum pump through the input circular RF flange and the circular downstream vacuum flange (3 and 6). The secondary vacuum chamber (4) serves two important purposes: 1) it prevents the occurrence of any virtual air pocket, created between cells clamped together, from leaking gas into the primary vacuum chamber; 2) it reduces the risk of contamination of the primary chamber caused by the welding process performed on the outer surfaces of the cavity. 


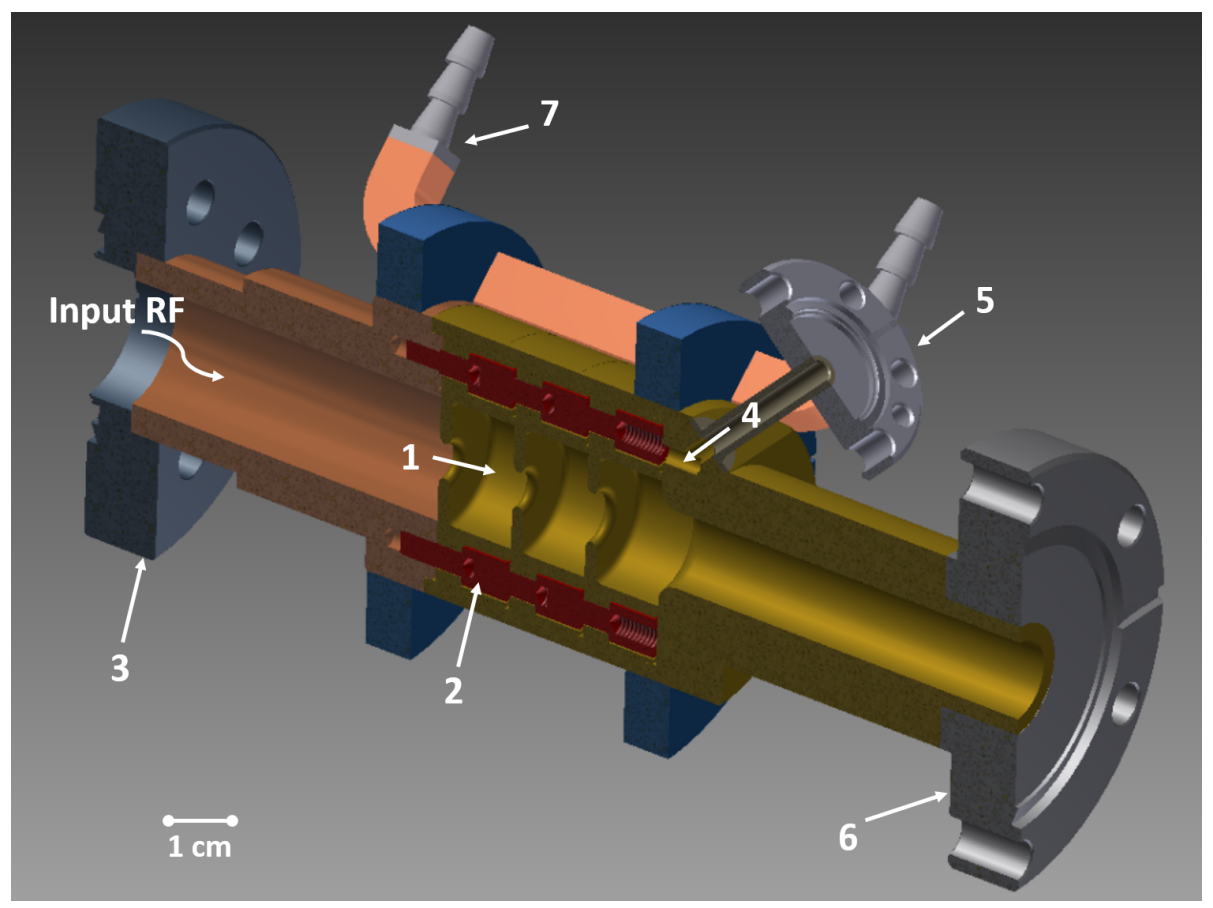

Figure 1. Solid model, one-half of the braze-free 3-cell cavity: 1. High-gradient cells, RF vacuum chamber; 2. Special screws for clamping; 3 . Input RF flange 4. secondary vacuum chamber; 5 . Vacuum flange for the secondary vacuum chamber; 6 . Downstream vacuum flange; 7 . Water cooling pipe.

\section{TIG welding process}

The Tungsten Inert Gas process exploits the heat generated by an electric discharge to join metallic parts. The weld area as well as the tungsten electrode itself are protected from oxidation by the inert gas exerted from the torch [17]. During the welding, the electric current is measured by two methods: the one that is set on the TIG machine and the effective one, which is read back on the screen during the melting process. In this contribution, we reference both of these currents. Various TIG welding tests were carried out on different cavities with different values of electric currents, and therefore different temperatures of the weld area. Hereby, we will always explicitly give the set current value as well as the effective current.

\section{Optimization of the welding tooth}

In figure 2, we show the 2-cell cavity structure machined for the purpose of this TIG welding test. On the left hand-side of the figure, the drawing shows the main features of the section of the cavity, in particular the tooth, characterized by a thickness and a depth, which is used for the welding. The tooth was machined on the outer edge of the structure with multiple purposes: localize the heating power from the TIG process and ensure that the temperature of the RF surfaces stays below the annealing values as well as to avoid temperature-related plastic deformations. The presence of the tooth practically allows for short welding time, which was typically less than two minutes per each turn. As expected, the smaller the tooth thickness, the smaller the welding current. On the 


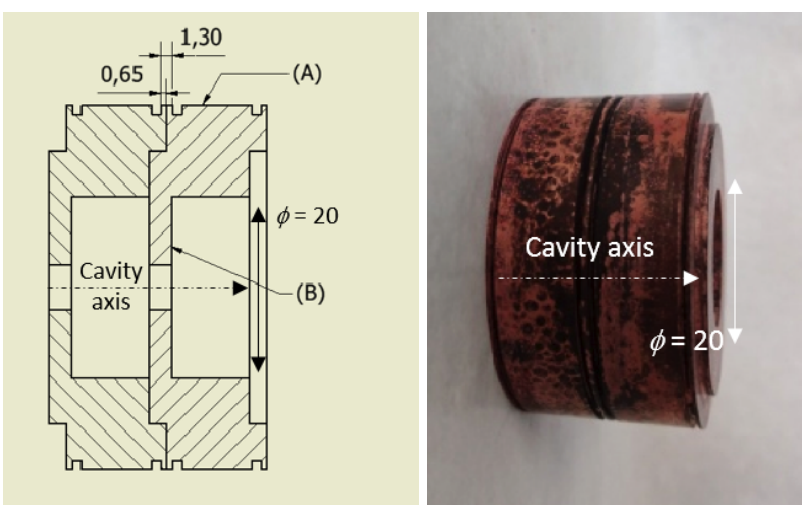

Figure 2. Left: drawing of the 2-cell setup for the TIG welding test (dimensions in mm) with a tooth thickness of $1.3 \mathrm{~mm}$. Right: photograph of the manufactured cells, after welding.

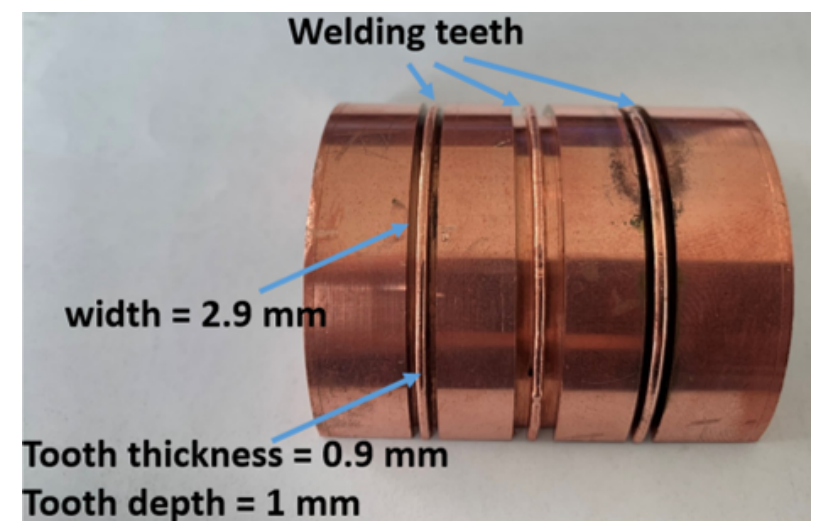

Figure 3. The copper structure pre-TIG process showing the machined teeth to localize the heat produced by the welding process.

other hand, a tooth that is too thin may not create a robust joint. The best compromise of the tooth thickness is the combination of the lowest possible current, suitable to achieve a mechanically stable and robust welding, and at the same time to obtain the ultra-high vacuum tightness. We have chosen a tooth thickness around $1 \mathrm{~mm}$. On the right-hand side of figure 2, it is shown the photograph of the side view of the manufactured cells after welding.

Various TIG welding tests were performed with different tooth thicknesses ranging from 0.8 $\mathrm{mm}$ up to $1.3 \mathrm{~mm}$ and with electric currents from $140 \mathrm{~A}$ to $200 \mathrm{~A}$. The optimal current value is correlated to the welding tooth thickness, since a thicker tooth needs a higher current in order to be melted. After all tests were performed, the optimal tooth thickness-welding current set that chosen for the final 3-cell prototype [14] was $0.9 \mathrm{~mm}$ and $160 \mathrm{~A}$, respectively.

In figure 3, we show the picture of the first 3-cell cavity prototype machined for testing the welding process. We indicate the thin teeth machined for the purposes mentioned above. Each tooths $1 \mathrm{~mm}$ deep and about $0.9 \mathrm{~mm}$ thick. The width of the spacing left on both sides of the tooth is $\sim 1 \mathrm{~mm}$.

In figure 4, we show the setup of the three thermocouples, which were attached to the cell main features in order to monitor the temperature during the TIG process. The first one to the cell iris, the second one to the welding tooth and the third one to the cell face (cell outer radius). 


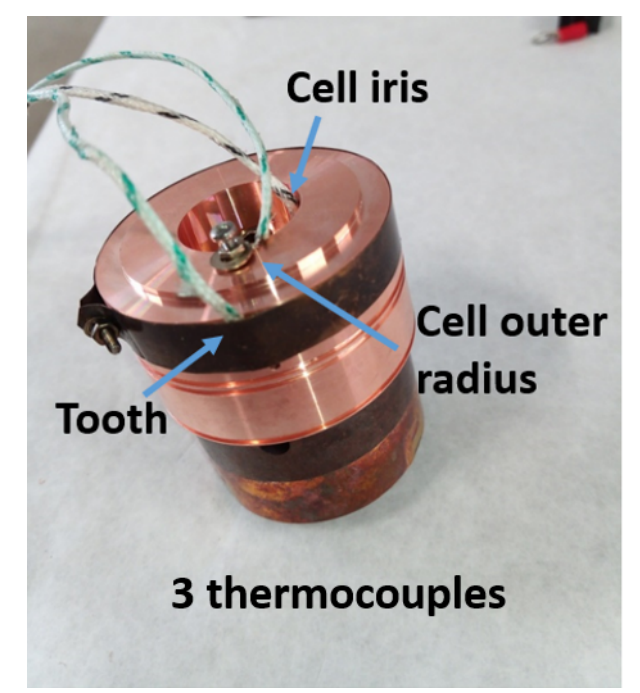

Figure 4. The three thermocouples wired and attached to the cell features: iris, face (outer radius) and tooth in order to monitor the temperature during the TIG process.

\section{The welding set-up}

The welding set-up is shown in figure 5 . The cavity to be welded is placed inside an aluminum cylinder in which a steady flow of argon, at a pressure of $\sim 1$ bar $(0.1 \mathrm{MPa})$, is used to avoid the oxidation of copper during the welding process. The torch enters through a circular hole in the cylinder side. In order to avoid oxidation of the cavity inner RF surfaces, two caps made out of Teflon were placed on either side of the structure. The Argon temperature at $\sim 1$ bar is equal to a few degrees Celsius and therefore it also improves the cooling of the copper. This is particularly important during the cooling down of the metal after the welding process since the risk of oxidation remains high until the copper temperature is above $80^{\circ} \mathrm{C}$. It has to be noticed that for the final structure that was sent to SLAC for RF high-power tests, argon was also flushed through the cavity in order to prevent oxidation of the high-field surfaces.

\section{Temperature reading}

In this section, we report the temperature readings from the thermocouples during the TIG welding process for three different cavity samples with three different values of tooth thickness and welding current. In table 1, the test results for the three cases (A, B and C) are summarized for clarity. The case $\mathrm{A}$, which is the first test performed, refers to the 3-cell cavity, as shown in figure 3, where the tooth thickness is $0.9 \mathrm{~mm}$. The tooth thickness for cases B and C are $1 \mathrm{~mm}$ and $0.8 \mathrm{~mm}$, respectively. For each case, the optimal electric current is also reported.

The plots of the temperature profiles during the welding process are similar for all cases. Here for the sake of simplicity we only show the readings for case A, which are given in figure 6 . The melting process takes about 70 seconds for one welding turn, as evident from the plots. In this case, we found that the optimal set electric current is $160 \mathrm{~A}$, while the effective current is about 133 A. For all thermocouples, which are placed on the tooth, the cell iris and the cell face, the copper 


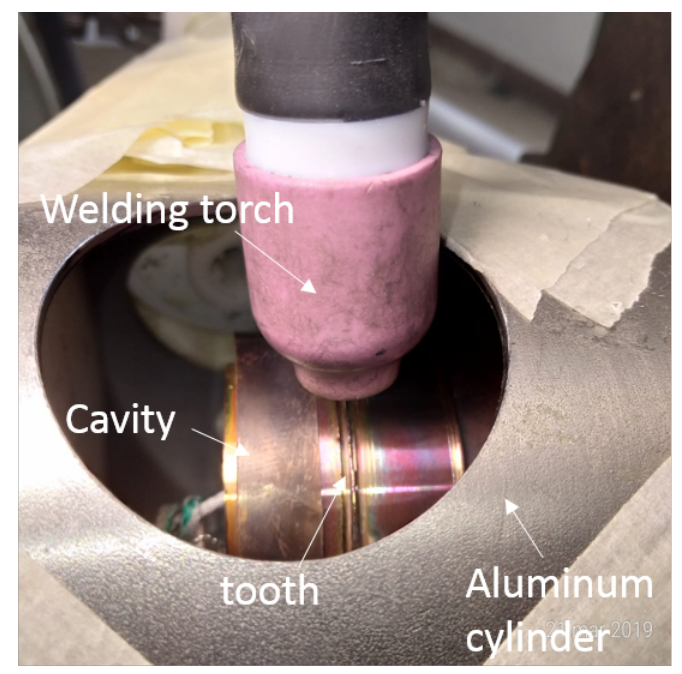

Figure 5. The TIG welding set-up: the cells to be welded are enclosed inside the aluminum cylinder and the torch enters through the circular hole in the cylinder. Argon is flushed inside the cylinder during the TIG process while cells rotate to melt the welding tooth.

Table 1. Results of the temperature monitoring during the TIG welding process for three different cases.

\begin{tabular}{|l|c|c|c|}
\hline & Case A & Case B & Case C \\
\hline Tooth Max Temperature $\left[{ }^{\circ} \mathrm{C}\right]$ & 321 & 414 & 298 \\
\hline Cell face Max Temperature $\left[{ }^{\circ} \mathrm{C}\right]$ & 310 & 400 & 249 \\
\hline Iris Max Temperature $\left[{ }^{\circ} \mathrm{C}\right]$ & 315 & 391 & 265 \\
\hline Welding time $[\mathrm{s}]$ & 70 & 80 & 80 \\
\hline Tooth thickness $[\mathrm{mm}]$ & 0.9 & 1 & 0.8 \\
\hline Set Current $[\mathrm{A}]$ & 160 & 180 & 140 \\
\hline Effective Current $[\mathrm{A}]$ & 133 & 149 & 117 \\
\hline
\end{tabular}

temperature rises almost linearly. These temperatures reach $321^{\circ} \mathrm{C}$ on the tooth, $310^{\circ} \mathrm{C}$ on the cell iris and $315^{\circ} \mathrm{C}$ on the cell face. After the torch is set off, the temperatures decay exponentially, as expected. It is important to underline here that the temperature on the RF surfaces, which comprise the iris and faces of the cell, remained well below the copper annealing temperature, which is $\sim 500^{\circ} \mathrm{C}$. Therefore, we expect that alterations of the physical and chemical properties of the copper are negligible or nil. In other words, the hardness of the material is preserved.

In the case $\mathrm{B}$, the tooth thickness is $1 \mathrm{~mm}$, the applied electric current is $180 \mathrm{~A}$ and the effective current on the welding tooth was $\sim 149 \mathrm{~A}$. The total time for one full welding turn was $\sim 80$ seconds. The temperature increases linearly up to $414^{\circ} \mathrm{C}$ on the tooth, $391^{\circ} \mathrm{C}$ on the cell iris and $400^{\circ} \mathrm{C}$ on the cell face. Again, it is important to underline that also for this welding current, the temperature on the RF surfaces, i.e. the iris and faces of the cell, remain below the copper annealing temperature.

For the case $\mathrm{C}$, the tooth thickness is $0.8 \mathrm{~mm}$ and the electric current applied is $140 \mathrm{~A}$ with an effective current on the welding tooth of $\sim 117 \mathrm{~A}$. The highest temperature is reached after $\sim 80$ seconds (one welding turn), during which the temperature linearly increases up to $298^{\circ} \mathrm{C}$ on the 


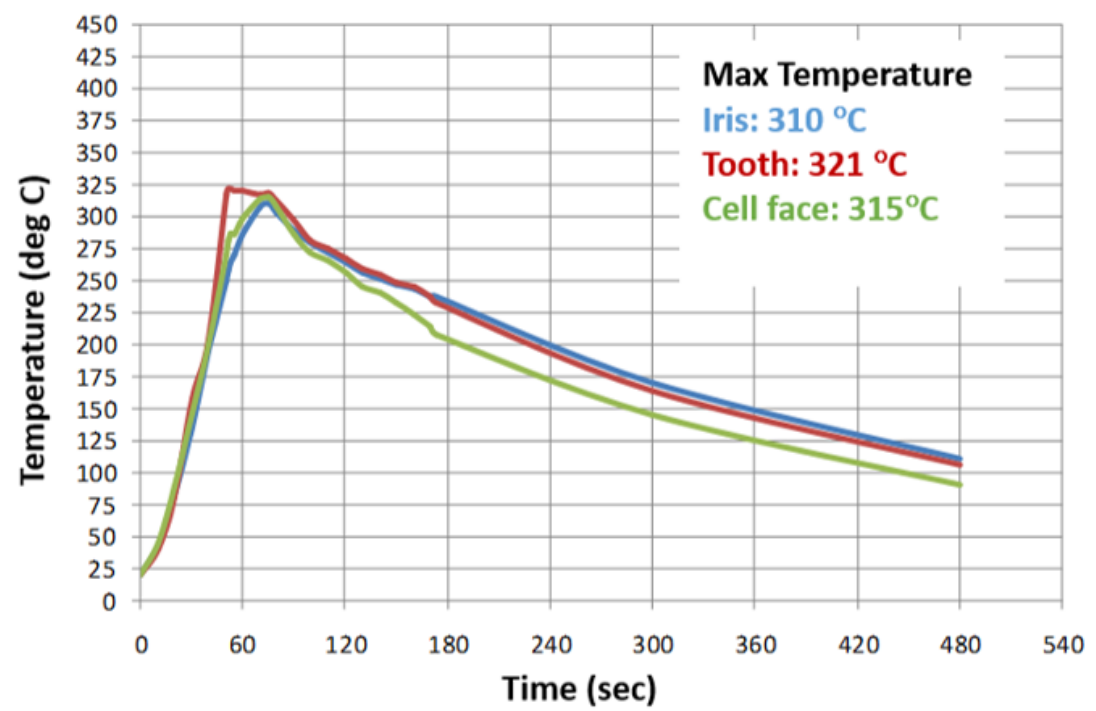

Figure 6. The temperature plots of the three thermocouples during one welding turn: the tooth thickness is $0.9 \mathrm{~mm}$ and the peak electric current $160 \mathrm{~A}$ (see text for more information).
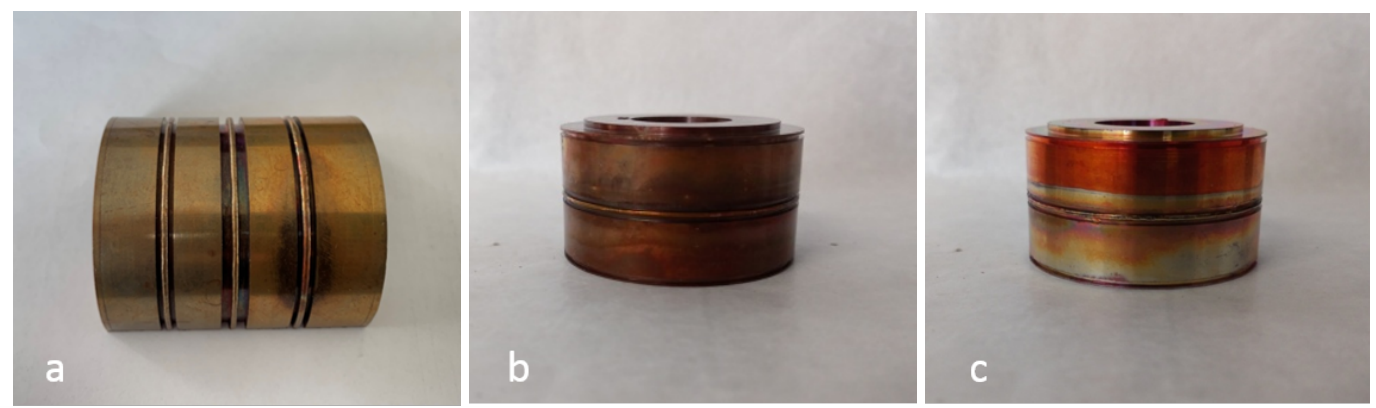

Figure 7. Ensemble of the photographs of the three cavity samples after the TIG welding process: (a) the 3-cell cavity of case A with a set electric current of $180 \mathrm{~A}$; (b) the 2-cell cavity of case B with a set electric current of $180 \mathrm{~A}$; (c) the 2-cell cavity of case $\mathrm{C}$ with a set electric current of $140 \mathrm{~A}$.

tooth, $265^{\circ} \mathrm{C}$ on the cell iris and $249^{\circ} \mathrm{C}$ on the cell face. Once more, it is important to notice that even in this lower current case, the temperature on the RF surfaces, which comprise iris and faces of the cell, remain always below the copper annealing temperature.

The ensemble of the photographs of the cavity samples after the TIG welding is shown in figure 7 ( $\mathrm{a}, \mathrm{b}$ and $\mathrm{c}$ ) for the three cases A, B and C, respectively. The picture in figure 7a is the 3-cell cavity of case A for which the set electric current was 160 A. Since we did not observe evident signatures of oxidation, this cavity geometry and temperature settings were identified as optimal. The 2-cell cavity after welding is shown in figure $7 \mathrm{~b}$ for the case $\mathrm{B}$, for which the set current was $180 \mathrm{~A}$. The copper oxidation is more evident in this case, as the darker brown areas of the surface point out. Nevertheless, no signs of oxidations were shown on the RF surfaces. In figure 7c, it is shown the photo of the 2-cell cavity of case $\mathrm{C}$ after welding with a set electric current of $140 \mathrm{~A}$. The surface oxidation is less evident in this case, and the copper cavity is characterized by a lighter colour on the outer surfaces. 

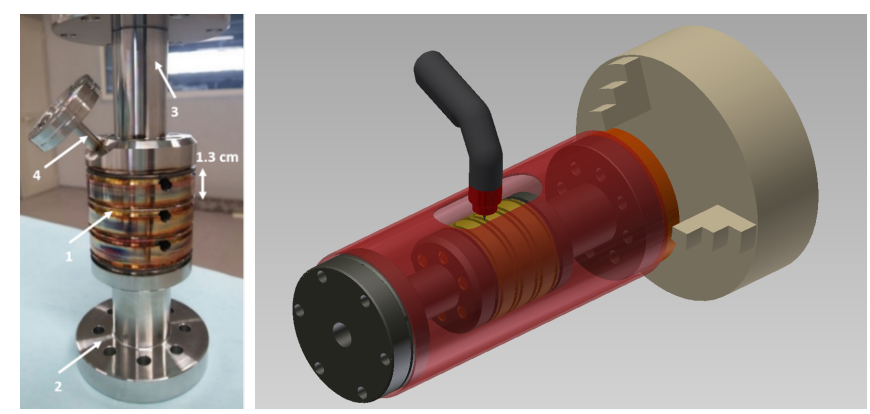

Figure 8. Left: photograph of the assembled 3-cell cavity after the TIG welding procedure. 1. welding joints; 2 . input RF flange; 3 . downstream vacuum flange; 4 . flange for pumping the secondary vacuum chamber. Right: rendering of the prototype setup of our TIG welding technology.

All tests performed demonstrated the possibility to obtain a vacuum-sealed structure with clean inner surfaces. It is worth mentioning there that the readings of all the thermocouples were consistent between each other during each test. However, since they might have moved among tests, an accurate comparison among the different welding processes was not possible.

\section{Welding of the final prototype}

The final prototype was machined by Comeb $s r l$ in Italy. It has been recently successfully tested at the SLAC laboratory at Stanford and the results of the high-power RF measurements are the subject of a forthcoming manuscript. On the right panel in figure 8 it is showed the $3 \mathrm{D}$ rendering of the prototype setup for TIG welding. In order to rotate the structure during the welding process one of the cavity flanges is held in place by the holder of the turning machine we used. Argon was flushed around and through the cavity, unlike the initial test samples, to avoid the oxidation of the RF surfaces.

\section{Conclusions}

Pure oxygen-free high-conductivity copper $\mathrm{C} 10100$ (Cu-OFE) is the most used material in many radio-frequency applications and, in particular, it is common for RF cavities, which are typically used for room temperature particle accelerator applications.

Recent studies have shown that harder materials offer better high-gradient performances than annealed ones. This has also been demonstrated in the case of cryogenic tests of copper cavities.

The technology of high-gradient accelerator components demands multiple requirements, such as high RF conductivity, ultra-high vacuum (UHV), no oxidation and no contamination of the material.

High-temperature brazing, either in vacuum or hydrogen atmosphere, and diffusion bonding are the widely methods used to fulfil these requirements. The temperatures, involved in these processes, significantly affect the mechanical properties of copper which becomes soft above $590^{\circ} \mathrm{C}$. In order to preserve the hardness of the material, we have developed a technology, which satisfies the above mentioned requirements without high temperature processing of the material. This method uses the TIG welding process, which provides structure integrity and UHV seal. Compared to brazing 
and diffusion bonding, TIG welding is a faster and cheaper technique. We optimized this process in order to keep the inner RF surfaces, which are later exposed to high fields, ultra-clean and below the material annealing values. As a consequence, the method does not affect the hardness of the heated material and it may allow us to achieve the maximum accelerating gradient in accelerating cavities made out of copper alloys.

\section{References}

[1] M. Ferrario et al., Eupraxia@SPARC_LAB design study towards a compact FEL facility at LNF, Nucl. Instrum. Meth. A 909 (2018) 134 [arXiv: 1801.08717].

[2] B. Spataro et al., High-power comparison among brazed, clamped and electroformed X-band cavities, Nucl. Instrum. Meth. A 657 (2011) 88.

[3] A. Grudiev, S. Calatroni and W. Wuensch, New local field quantity describing the high-gradient limit of accelerating structures, Phys. Rev. ST Accel. Beams 12 (2009) 102001.

[4] V.A. Dolgashev et al., Status of high power tests of normal conducting single-cell standing wave structures, in the proceedings of the $1^{\text {st }}$ International Particle Accelerator Conference (IPAC'10), May 23-28, Kyoto, Japan (2010).

[5] V.A. Dolgashev, High gradient, X-band and above, metallic RF structures, in the proceedings of the $2^{\text {nd }}$ European Advanced Accelerator Concepts Workshop (AAC2015), September 13-19, La Biodola, Italy (2015).

[6] H. Zha, A. Grudiev and V.A. Dolgashev, RF design of the CLIC structure prototype optimized for manufacturing from two halves, in the proceedings of the $6^{\text {th }}$ International Particle Accelerator Conference (IPAC'15), May 3-8, Richmond, U.S.A. (2015).

[7] E.A. Nanni et al., Toward high-power high-gradient testing of mm-wave standing-wave accelerating structures, in the proceedings of the $9^{\text {th }}$ International Particle Accelerator Conference (IPAC'18), April 29-May 4, Vancouver, Canada. (2018).

[8] V.A. Dolgashev et al., RF breakdown measurements in electron beam driven $200 \mathrm{GHz}$ copper and copper-silver accelerating structures, Phys. Rev. Accel. Beams 19 (2016) 111301.

[9] L. Laurent et al., Experimental study of rf pulsed heating, Phys. Rev. ST Accel. Beams 14 (2011) 041001.

[10] Korsbäck et al., Vacuum electrical breakdown conditioning study in a parallel plate electrode pulsed DC system, arXiv: 1905.03996.

[11] I. Profatilova, L.M. Morales and N. Pienimaki, High-gradient test results from dc system: conditioning and pulse length dependence, talk given at Pulsed dc system results, August 17, CERN, Switzerland (2016).

[12] A.D. Cahill et al., High gradient experiments with X-band cryogenic copper accelerating cavities, Phys. Rev. Accel. Beams 21 (2018) 102002.

[13] https://www.copper.org/resources/properties/144_8/

[14] V.A. Dolgashev, L. Faillace, B. Spataro and R. Bonifazi, Innovative compact braze-free accelerating cavity, 2018 JINST 13 P09017.

[15] V.A. Dolgashev et al., RF breakdown tests of mm-wave metallic accelerating structures, Phys. Rev. Accel. Beams 19 (2016) 011301. 
[16] H. Zha and A. Grudiev, Design of the Compact Linear Collider main linac accelerating structure made from two halves, Phys. Rev. Accel. Beams 20 (2017) 042001.

[17] https://en.wikipedia.org/wiki/Gas_tungsten_arc_welding.

[18] A.S. Pohjonen et al., Dislocation nucleation from near surface void under static tensile stress in Cu, J. Appl. Phys. 110 (2011) 023509.

[19] D. Yadav and R. Bauri, Effect of friction stir processing on microstructure and mechanical properties of aluminium, Mat. Sci. Eng. A 539 (2012) 85.

[20] D. Lohwasser, and Z. Chen, Friction stir welding: from basics to applications, Woodhead Publishing, Cambridge U.K. (2010).

[21] D.G. Brandon, W.D. Kaplan, Microstructural characterization of materials, Wiley, New York U.S.A. (1999).

[22] R.A. Matula, Electrical resistivity of copper, gold, palladium, and silver, J. Phys. Chem. Ref. Data 8 (1979) 1147.

[23] R.P. Reed and R.P. Mikesell, Low temperature mechanical properties of copper and selected copper alloys, National Bureau of Standards, Boulder, U.S.A. (1967).

[24] J.B. Rosenzweig et al., Next generation high brightness electron beams from ultra-high field cryogenic radiofrequency photocathode sources, Phys. Rev. Accel. Beams 22 (2019) 023403 [arXiv: 1603.01657].

[25] A. Hassanein et al., Effects of surface damage on RF cavity operation, Phys. Rev. ST Accel. Beams 9 (2006) 062001.

[26] Z. Cheng et al., Extra strengthening and work hardening in gradient nanotwinned metals, Science 362 (2018) 6414.

[27] M.A. Meyers and K.K. Chawla, Mechanical behaviour of materials, Englewood Cliffs, U.S.A. (1999). 